\title{
Erratum to: Evolving Approaches to Metastatic Breast Cancer Patients Pre-treated with Anthracycline and Taxane
}

\author{
Shigehira Saji
}

Published online: 27 August 2013

(C) Springer International Publishing Switzerland 2013

\section{Erratum to: BioDrugs \\ DOI 10.1007/s40259-013-0038-1}

A Published-Ahead-of-Print version of this article was made available online on 9 May 2013 at http://link. springer.com/journal/40259/onlineFirst/page/1.

Errors were subsequently identified in that version of the article, and the following corrections should be noted.

Page 3, section 3.2.1, column 1, 1st paragraph, lines 9-13:

The following phrase which reads:

"In the per-protocol population of the smaller study $(n=87)$, eribulin had an overall response rate of $11.5 \%$, whereas patients in the larger study had an overall response rate of $9.3 \%$; in both studies, all responses were considered partial $[27,28] . "$

should read:

"In the per-protocol population of the smaller study $(n=87)$, eribulin had an overall response rate of $11.5 \%$, whereas patients in the larger study who met key inclusion criteria (eligible patients, $n=269$ ) had an overall response rate of $9.3 \%$; in both studies, all responses were considered partial [27, 28]."

Page 5, section 3.2.2, column 1, 3rd paragraph, lines 4-7:

The following phrase which reads:

"Patients were required to have previously received at most three chemotherapy regimens (at most two for

The online version of the original article can be found under doi:10.1007/s40259-013-0038-1.

\section{S. Saji $(\square)$}

Department of Target Therapy Oncology, Kyoto University

Graduate School of Medicine, 54 Shogoin Kawahara-cho,

Sakyo-ku, Kyoto 606-8507, Japan

e-mail: ss-saji@wa2.so-net.ne.jp advanced disease) with each regimen including an anthracycline or a taxane."

should read:

"Patients were required to have previously received anthracycline and taxane therapy, and at most three chemotherapy regimens (at most two for advanced disease)."

Page 5, section 3.2.2, column 1, 4th paragraph, line 1:

The following phrase which reads:

"Like the EMBRACE study, eribulin increased the median overall survival of patients compared with capecitabine (15.9 vs 14.5 months; HR $0.88,95 \%$ CI 0.77 , $1.00 ; p=0.056$; Table 4), although this difference was not statistically significant."

should read:

"Eribulin increased the median overall survival of patients compared with capecitabine (15.9 vs 14.5 months; HR $0.88,95 \%$ CI $0.77,1.00 ; p=0.056$; Table 4), although this difference was not statistically significant."

Page 6, section 3.3, column 1, 4th paragraph, lines 3-9 and column 2, lines 10-14:

The following phrase which reads:

"Adverse events were reported in $94.1 \%$ of patients receiving eribulin and $90.5 \%$ of patients receiving capecitabine; 17.5 and $21.1 \%$ of patients reported serious adverse events [31]. More patients receiving eribulin had neutropenia (54 vs $16 \%$ ) and leukopenia (31 vs $10 \%$ ); however, the incidence of anaemia, thrombocytopenia and febrile neutropenia was similar between treatment groups [31]. Other common adverse events reported in patients receiving eribulin included alopecia (35\%), nausea (22\%), fatigue (17\%) and asthenia $(15 \%)$. Peripheral sensory neuropathy was observed in $13 \%$ of patients (grade 3, $4 \%$ of patients; no grade 4) [31]." should read:

"More patients receiving eribulin had neutropenia (54 vs $16 \%$ ) and leukopenia (31 vs $10 \%$ ); other common 
adverse events reported in patients receiving eribulin included alopecia (35\%) and nausea (22\%) [31]."

Page 9, column 2, reference 31

Reference should be replaced with:

"Kaufman PA, Awada A, Twelves C, Yelle L, Perez EA, Wanders J, et al. A phase III, open-label, randomized, multicenter study of eribulin mesylate versus capecitabine in patients with locally advanced or metastatic breast cancer previously treated with anthracyclines and taxanes. Cancer Res 2012; 72 (Suppl. 24): Abs S6-6."

Page 10, column 2, references 50, 51 and 52

References should be replaced with:

50. "Vahdat L, Schwartzberg L, Glück S, Rege J, Liao J, Cox D, et al. Results of a phase 2, multicenter, single-arm study of eribulin mesylate as first-line therapy for locally recurrent or metastatic HER2-negative breast cancer. Cancer Res 2012; 72 (Suppl. 24): Abs P1-12-02."

51. "Vahdat L, Schwartzberg L, Wilks S, Rege J, Liao J, Cox D, et al. Eribulin mesylate + trastuzumab as first-line therapy for locally recurrent or metastatic HER2-positive breast cancer: results from a phase 2, multicenter, singlearm study. Cancer Res 2012; 72 (Suppl. 24): Abs P5-2004."

52. "Traina TA, Hudis C, Fornier M, Lake D, Lehman $\mathrm{R}$, Berkowitz AP, et al. Adjuvant treatment of early-stage breast cancer with eribulin mesylate following dose-dense doxorubicin and cyclophosphamide: preliminary results from a phase 2, single-arm feasibility study . Cancer Res 2012; 72 (Suppl. 24): Abs P1-13-11.”

Page 10, column 2

The following reference should be inserted

"55. Pharmaceuticals and Medical Devices Agency, Japan. Halaven $1 \mathrm{mg}$ Rinsyo Gaiyou, section 2.7.3-5, page 28 (Japanese only) [online]. Available from URL: http:// www.info.pmda.go.jp/shinyaku/P201100077/index.html [Accessed 2013 Jul 15]."

Page 4, Table 3 should be replaced with (bolded text is amended):

Page 6, Table 5 should be replaced with (bolded text is amended):

Table 3 Phase II studies of eribulin in patients with metastatic breast cancer who have previously received an anthracycline and taxane

\begin{tabular}{|c|c|c|c|}
\hline$n$ & $\begin{array}{l}201[27] \\
87 \text { (per protocol population) }\end{array}$ & $\begin{array}{l}211 \text { [28] } \\
269 \text { (eligible population) }\end{array}$ & $\begin{array}{l}221[29] \\
80 \text { (eligible population) }\end{array}$ \\
\hline Prior chemotherapy & $\begin{array}{l}\text { Any prior regimen of chemotherapy } \\
\text { with } \mathrm{A} \text { and } \mathrm{T} \text { (median 4) }\end{array}$ & $\begin{array}{l}2-5 \text { prior regimens of chemotherapy } \\
\text { with } \mathrm{A}, \mathrm{T} \text { and CAP (median } 4)\end{array}$ & $\begin{array}{l}\leq 3 \text { prior regimens of chemotherapy } \\
\text { including } \mathrm{A} \text { and } \mathrm{T} \text { (median } 3 \text { ) }\end{array}$ \\
\hline Dosing schedule & $\begin{array}{l}1.4 \mathrm{mg} / \mathrm{m}^{2} \text { IV inf } \\
\mathrm{d} 1+8+15 \mathrm{q} 4 \mathrm{w} \\
1.4 \mathrm{mg} / \mathrm{m}^{2} \text { IV inf } \mathrm{d} 1+8 \\
\mathrm{q} 3 \mathrm{w}\end{array}$ & $\begin{array}{l}1.4 \mathrm{mg} / \mathrm{m}^{2} \mathrm{IV} \text { inf } \mathrm{d} 1+8 \\
\mathrm{q} 3 \mathrm{w}\end{array}$ & $\begin{array}{l}1.4 \mathrm{mg} / \mathrm{m}^{2} \mathrm{IV} \text { inf } \mathrm{d} 1+8 \\
\mathrm{q} 3 \mathrm{w}\end{array}$ \\
\hline \multicolumn{4}{|l|}{$\begin{array}{l}\text { Tumour response } \\
\text { (independent review) }\end{array}$} \\
\hline PR $(\%)$ & $\begin{array}{l}11.5 \text { [total] } \\
10.2 \text { [q4w cohort] } \\
14.3 \text { [q3w cohort] }\end{array}$ & 9.3 & 21.3 \\
\hline $\mathrm{SD}(\%)$ & $\begin{array}{l}\text { 42.5 [total] } \\
\text { 35.6 [q4w cohort] } \\
\mathbf{5 7 . 1}[q 3 w \text { cohort }]\end{array}$ & 46.5 & 37.5 \\
\hline $\mathrm{ORR}^{\mathrm{a}}(\%)$ & $\begin{array}{l}11.5 \text { [total] } \\
10.2 \text { [q4w cohort] } \\
14.3 \text { [q3w cohort] }\end{array}$ & 9.3 & 21.3 \\
\hline $\mathrm{CBR}^{\mathrm{b}}(\%)$ & $\begin{array}{l}17.2 \text { [total] } \\
11.9 \text { [q4w cohort] } \\
28.6 \text { [q3w cohort] }\end{array}$ & 17.1 & 27.5 \\
\hline $\begin{array}{l}\text { Median duration of } \\
\text { response (months) }\end{array}$ & 5.6 & 4.1 & 3.9 \\
\hline Median PFS (months) & 2.6 & 2.6 & 3.7 \\
\hline Median OS (months) & 9.0 & 10.4 & 11.1 \\
\hline
\end{tabular}

$A$ anthracycline, $C A P$ capecitabine, $C B R$ clinical benefit rate, $d$ day, $I V$ inf intravenous infusion, ORR objective response rate, $O S$ overall survival, $P F S$ progression-free survival, $P R$ partial response, $q X w$ every $X$ weeks, $S D$ stable disease, $T$ taxane

${ }^{\text {a }}$ Objective response rate $=$ complete response + partial response

${ }^{\mathrm{b}}$ Clinical benefit rate $=$ complete response + partial response + stable disease $\geq 6$ months 
Table 5 Overall survival in the phase III studies of eribulin by human epidermal growth factor receptor 2 (HER2) and oestrogen receptor (ER) status

\begin{tabular}{|c|c|c|c|c|c|c|}
\hline & \multicolumn{3}{|c|}{305 (EMBRACE) $[30, \mathbf{5 5}]$} & \multicolumn{3}{|c|}{$301[31,32]$} \\
\hline & \multicolumn{2}{|c|}{ OS (months) } & \multirow[t]{2}{*}{ HR $(95 \%$ CI $)$} & \multicolumn{2}{|c|}{ OS (months) } & \multirow[t]{2}{*}{$\mathrm{HR}(95 \% \mathrm{CI})$} \\
\hline & Eribulin & TPC & & Eribulin & CAP & \\
\hline Total & 13.1 & 10.6 & $0.81(0.66,0.99)$ & 15.9 & 14.5 & $0.88(0.77,1.00)$ \\
\hline HER2 + & $11.3^{\mathrm{a}}$ & $9.1^{\mathrm{a}}$ & $0.76(0.47,1.24)$ & 14.3 & 17.1 & $0.97(0.69,1.36)$ \\
\hline HER 2- & $13.2^{\mathrm{a}}$ & $10.5^{\mathrm{a}}$ & $0.81(0.64,1.02)$ & 15.9 & 13.5 & $0.84(0.72,0.98)$ \\
\hline ER+ & $13.8^{\mathrm{a}}$ & $11.4^{\mathrm{a}}$ & $0.81(0.63,1.04)$ & 18.2 & 16.8 & $0.90(0.74,1.09)$ \\
\hline ER- & $10.2^{\mathrm{a}}$ & $7.8^{\mathrm{a}}$ & $0.78(0.54,1.13)$ & 14.4 & 10.5 & $0.78(0.64,0.96)$ \\
\hline $\mathrm{TN}$ & $9.5^{\mathrm{a}}$ & $7.0^{\mathrm{a}}$ & $0.71(0.46,1.10)$ & 14.4 & 9.4 & $0.70(0.55,0.91)$ \\
\hline
\end{tabular}

$\overline{C A P}$ capecitabine, ER oestrogen receptor, HER2 human epidermal growth factor receptor 2, $T N$ triple negative, $T P C$ treatment of physician's choice

a Values calculated from data available from the Pharmaceuticals and Medical Devices Agency, Japan. Halaven 1 mg Rinsyo Gaiyou [55] 